\title{
Research Article \\ Computation of All Stabilizing PID Gain for Second-Order Delay System
}

\author{
Rihem Farkh, Kaouther Laabidi, and Mekki Ksouri \\ Research Unit on Systems Analysis and Control, National School of Engineering of Tunis, BP, 37, \\ Le Belvédère, 1002 Tunis, Tunisia \\ Correspondence should be addressed to Rihem Farkh, rihem.farkh@enit.rnu.tn
}

Received 2 April 2009; Revised 19 June 2009; Accepted 15 July 2009

Recommended by Ji Huan He

The problem of stabilizing a second-order delay system using classical proportional-integralderivative (PID) controller is considered. An extension of the Hermite-Biehler theorem, which is applicable to quasipolynomials, is used to seek the set of complete stabilizing PID parameters. The range of admissible proportional gains is determined in closed form. For each proportional gain, the stabilizing set in the space of the integral and derivative gains is shown to be either a trapezoid or a triangle.

Copyright (C) 2009 Rihem Farkh et al. This is an open access article distributed under the Creative Commons Attribution License, which permits unrestricted use, distribution, and reproduction in any medium, provided the original work is properly cited.

\section{Introduction}

Dead times are often encountered in various engineering systems and industry processes such as electrical and communication network, chemical process, turbojet engine, nuclear reactor, and hydraulic system. In fact, delays are caused by many phenomena like the time required to transport mass, energy or information, the time processing for sensors, the time needed for controllers to execute a complicated algorithm control, and the accumulation of time lags in a number of simple plants connected in series [1]. Delay makes system analysis and control design much more complex which is explained as follows. First, the effect of the control action takes some time to be felt in the controlled variable. Second, the control action that is applied based on the actual error tries to correct a situation that originated previously. Finally, in the frequency domain, the time delay introduces an extra decrease in the systems phase, which may cause instability [1,2].

Today, proportional-integral (PI) and proportional-integral-derivative (PID) controller types are the most widely used control strategy. It is estimated that over $90 \%$ of process control applications employ PID control thanks to its essential functionality and structural 
simplicity [3]. Since the minimal requirement for PID controller is to guarantee the system stability. It is desirable to know the complete set of stabilizing PID parameters before tuning and design. Surveys have reported that poor tuning, configuration errors, traditional and empirical techniques such as Ziegler Nichols or Cohen-Coon tuning methods are found in the industrial application of PID [4]. A great deal of academic and industrial effort has focused on improving PID control in the areas of tuning rules to decrease the rising gap between engineering practice and control theory. Recent studies have made use of the generalization of Hermite-Biehler theorem to compute the set of all stabilizing PID controllers for a given plant [5]. Since almost all plants encountered in process control contain time delays, so computing the complete set of PID controllers that stabilize time delay system is of considerable importance. A robust PI/PID design via numerical optimization for delay processes was proposed in [6]. Padma Sree et al. [7] propose a PI/PID controllers design for first-order delay system by extracting the coefficients of the numerator and denominator of the closed-loop transfer function. In the work presented in [8], the authors have developed a graphical approach which is based on D-partition method [9], the borders of absolute and relative stability regions are described in the parameter space. A unified approach and Delta operator are used to obtain unified stability boundaries for PI, PD, and PID controller for an arbitrary order delay system; where the boundaries can be found when only the frequency response and not the parameters of the plant are known [10]. Eriksson and Johansson [11] consider a design of PID controller for system with varying time delay using multi-objectives' optimization. Neural network has been widely applied for nonlinear dynamical system identification via PID controller [12]. Zhang et al. [13] present an intelligent nonlinear PID controller based on the recurrent neural networks (RNNs) to control multivariable plants by using two predictive control schemes. In [14, 15], the authors propose a new PID neural networks (PIDNN) which is a dynamic multilayer network based on P, I, and D neurons. This PIDNN is used to control multivariable plants [15] and has also the ability to control time delay system [14]. In [16], Roy and Iqbal have explored PID tuning of first-order delay system using a first-order Padé approximation and the Hermite-Biehler stabilization framework. The set's characterization of all stabilizing PI/PID parameters using a version of the Hermit-Biehler theorem for first-order delay system is presented in [5, 17-20]. However, these results are not applicable to the second-order delay system. Although, the PID stabilization for a given time delay plant has been developed [21] using the generalized Nyquist criterion, the stabilizing set of the proportional gain cannot be found. In [22-24], the characterization of the set of all stabilizing P/PI/PID parameters is given by using the Hermit-Biehler Theorem for polynomials for a class of time delay system which verify the interlacing property at high frequencies. This method, however, is complex and there are difficulties to achieve the stabilizing range of proportional parameter. As shown in [23, Example 2], this latter approach does not determine the precise range of proportional gain which is larger than the exact stabilizing range given by [18]. Furthermore, a known stabilizing value of $\left(K_{p}, K_{i}, K_{d}\right)$ is first determined using Nyquist criterion, then the entire stability region is established. In our work, the stabilizing problem of PID controller for second order delay system is analyzed using the extended HermitBiehler theorem for quasi-polynomials. The exact range of stabilizing proportional gain is first determined. The set of stabilizing integral and derivative constant values are then derived. Our algorithm is more simple and faster in time computing than the other presented in [24]. 


\section{Preliminary Results for Analyzing Time Delay System}

Several problems in process control engineering are related to the presence of delays. These delays intervene in dynamic models whose characteristic equations are of the following form $[5,25]$

$$
\delta(s)=d(s)+e^{-L_{1} s} n_{1}(s)+e^{-L_{2} s} n_{2}(s)+\cdots+e^{-L_{m} s} n_{m}(s),
$$

where: $d(s)$ and $n_{i}(s)$ are polynomials with real coefficients and $L_{i}$ represent time delays. These characteristic equations are recognized as quasi-polynomials. Under the following assumptions:

$\left(A_{1}\right) \operatorname{deg}(d(s))=n, \operatorname{deg}\left(n_{i}(s)\right)<n$, for $i=1,2, \ldots, m$,

$\left(A_{2}\right) L_{1}<L_{2}<\cdots<L_{m}$.

One can consider the quasi-polynomials $\delta^{*}(s)$ described by

$$
\begin{gathered}
\delta^{*}(s)=e^{s L_{m}} \delta(s), \\
\delta^{*}(s)=e^{s L_{m}} d(s)+e^{s\left(L_{m}-L_{1}\right)} n_{1}(s)+e^{s\left(L_{m}-L_{2}\right)} n_{2}(s)+\cdots+n_{m}(s) .
\end{gathered}
$$

The zeros of $\delta(s)$ are identical to those of $\delta^{*}(s)$ since $e^{s L_{m}}$ does not have any finite zeros in the complex plane. However, the quasi-polynomial $\delta^{*}(s)$ has a principal term since the coefficient of the term containing the highest powers of $s$ and $e^{s}$ is nonzero. If $\delta^{*}(s)$ does not have a principal term, then it has an infinity roots with positive real parts [5].

The stability of the system with the characteristic equation (2.1) is equivalent to the condition that all the zeros of $\delta^{*}(s)$ must be in the open left half of the complex plan. We said that $\delta^{*}(s)$ is Hurwitz or is stable. The following theorem gives a necessary and sufficient condition for the stability of $\delta^{*}(s)$.

Theorem 2.1 (see [5]). Let $\delta^{*}(s)$ be given by (2.2), and write

$$
\delta^{*}(j \omega)=\delta_{r}(\omega)+j \delta_{i}(\omega)
$$

where $\delta_{r}(\omega)$ and $\delta_{i}(\omega)$ represent, respectively, the real and imaginary parts of $\delta^{*}(j \omega)$.

Under conditions $\left(A_{1}\right)$ and $\left(A_{2}\right), \delta^{*}(s)$ is stable if and only if

(1) $\delta_{r}(\omega)$ and $\delta_{i}(\omega)$ have only simple, real roots and these interlace,

(2) $\delta^{\prime}{ }_{i}\left(\omega_{0}\right) \delta_{r}\left(\omega_{0}\right)-\delta_{i}\left(\omega_{0}\right) \delta^{\prime}{ }_{r}\left(\omega_{0}\right)>0$ for some $\omega_{0}$ in $[-\infty,+\infty]$,

where $\delta_{i}^{\prime}(\omega)$ and $\delta_{r}^{\prime}(\omega)$ denote the first derivative with respect to $\omega$ of $\delta_{r}(\omega)$ and $\delta_{i}(\omega)$, respectively.

A crucial stage in the application of the precedent theorem is to make sure that $\delta_{r}(\omega)$ and $\delta_{i}(\omega)$ have only real roots. Such a property can be checked while using the following theorem. 
Theorem 2.2 (see [5]). Let $M$ and $N$ designate the highest powers of $s$ and $e^{s}$ which appear in $\delta^{*}(s)$. Let $\eta$ be an appropriate constant such that the coefficient of terms of highest degree in $\delta_{r}(\omega)$ and $\delta_{i}(\omega)$ do not vanish at $\omega=\eta$. Then a necessary and sufficient condition that $\delta_{r}(\omega)$ and $\delta_{i}(\omega)$ have only real roots is that in each of the intervals $-2 l \pi+\eta<\omega<2 l \pi+\eta, l=l_{0}, l_{0}+1, l_{0}+2 \cdots \delta_{r}(\omega)$ or $\delta_{i}(\omega)$ have exactly $4 l N+M$ real roots for a sufficiently large $l_{0}$.

\section{PID Control for Second Order Delay System}

A second order system with delay can be mathematically expressed by a transfer function having the following form:

$$
G(s)=\frac{K}{s^{2}+a_{1} s+a_{0}} e^{-L s}
$$

where $K$ is the static gain of the plant, $L$ is the time delay, and $a_{0}$ and $a_{1}$ are the plant parameter's which are always positive. The characteristic equation of the closed-loop system is given by

$$
\delta(s)=K\left(K_{i}+K_{p} s+K_{d} s^{2}\right) e^{-L s}+\left(s^{2}+a_{1} s+a_{0}\right) s
$$

we deduce the quasi-polynomial $\delta^{*}(s)$ :

$$
\delta^{*}(s)=e^{L s} \delta(s)=K\left(K_{i}+K_{p} s+K_{d} s^{2}\right)+s\left(s^{2}+a_{1} s+a_{0}\right) e^{L s},
$$

by replacing $s$ by $j \omega$, we get

$$
\delta^{*}(j \omega)=\delta_{r}(\omega)+j \delta_{i}(\omega)
$$

with:

$$
\begin{aligned}
& \delta_{r}(\omega)=K K_{i}-K K_{d} \omega+\left(\omega^{3}-a_{0} \omega\right) \sin (L \omega)-a_{1} \omega^{2} \cos (L \omega), \\
& \delta_{i}(\omega)=w\left[K K_{p}+\left(a_{0}-\omega^{2}\right) \cos (L \omega)-a_{1} \omega \sin (L \omega)\right] .
\end{aligned}
$$

Clearly, the parameters $K_{i}$ and $K_{d}$ only affect the real part of $\delta^{*}(j \omega)$ whereas the parameter $K_{p}$ affects the imaginary part.

Let's put $z=L \omega$, we get

$$
\begin{aligned}
& \delta_{r}(z)=K K_{i}-K K_{d} \frac{z^{2}}{L^{2}}+\sin (z)\left(\frac{z^{3}}{L^{3}}-a_{0} \frac{z}{L}\right)-a_{1} \frac{z^{2}}{L^{2}} \cos (z), \\
& \delta_{i}(z)=\frac{z}{L}\left(K K_{p}+\cos (z)\left(a_{0}-\frac{z^{2}}{L^{2}}\right)-a_{1} \frac{z}{L} \sin (z)\right) .
\end{aligned}
$$


The application of the second condition of Theorem 2.1, led us to

$$
E\left(z_{0}\right)=\delta_{i}^{\prime}\left(z_{0}\right) \delta_{r}\left(z_{0}\right)-\delta_{i}\left(z_{0}\right) \delta_{r}^{\prime}\left(z_{0}\right)>0
$$

from (3.6) we have

$$
\delta_{i}^{\prime}(z)=\frac{K K_{p}}{L}-\sin (z)\left(a_{0}+\frac{2 a_{1} z}{L^{2}}-\frac{z^{3}}{L^{3}}\right)+\cos (z)\left(\frac{a_{0}}{L}-\frac{3 z^{2}}{L^{3}}-a_{1} \frac{z^{2}}{L^{2}}\right)
$$

for $z_{0}=0$ (a value that annul $\delta_{i}(z)$ ), and we get:

$$
E\left(z_{0}\right)=\delta_{i}^{\prime}\left(z_{0}\right) \delta_{r}\left(z_{0}\right)=\left(\frac{K K_{p}+a_{0}}{L}\right) K K_{i}>0
$$

which implies $K_{p}>-a_{0} / K$ since $K>0$ and $K_{i}>0$.

We pass to the verification of the interlacing condition of $\delta_{r}(z)$ and $\delta_{i}(z)$ roots. For such purpose, we are going to determine the roots from the imaginary part, which is translated by the following relation:

$$
\begin{aligned}
\delta_{i}(z)=0 & \Longrightarrow z=0 \quad \text { or } \quad K K_{p}+\cos (z)\left(a_{0}-\frac{z^{2}}{L^{2}}\right)-a_{1} \frac{z}{L} \sin (z)=0 \\
& \Longrightarrow z=0 \quad \text { or } \quad K K_{p}+\cos (z)\left(a_{0}-\frac{z^{2}}{L^{2}}\right)=a_{1} \frac{z}{L} \sin (z), \\
& \Longrightarrow z=0 \quad \text { or } \quad f(z)=g(z) .
\end{aligned}
$$

We notice the $z_{0}=0$ is a trivial root of the imaginary part. The others are difficult to solve analytically. However, this can be made graphically. The following two cases are presented.

Case $1\left(K_{p}>-a_{0} / K\right)$. In this case, we graph the curves of $f(z)$ and of $g(z)$ which are presented in Figure 1.

$$
\begin{array}{cc}
z_{1} \in[0, \pi / 2], & z_{2} \in[\pi / 2, \pi], \\
z_{3} \in[3 \pi / 2,2 \pi], & z_{4} \in[5 \pi / 2,3 \pi], \\
z_{5} \in[7 \pi / 2,4 \pi], & z_{6} \in[9 \pi / 2,5 \pi],
\end{array}
$$




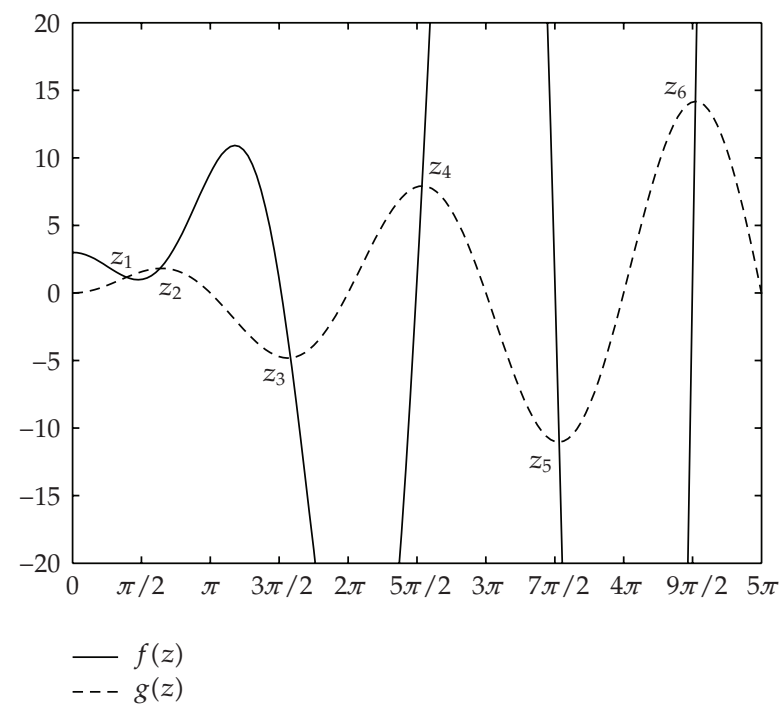

Figure 1: Representation of the curves of $f(z)$ and of $g(z)\left(K=L=a_{1}=1, a_{0}=2, K_{p}=1.3\right.$, and $\left.K_{u}=1.5884\right)\left(\right.$ Case: $\left.K_{p}<K_{u}\right)$.

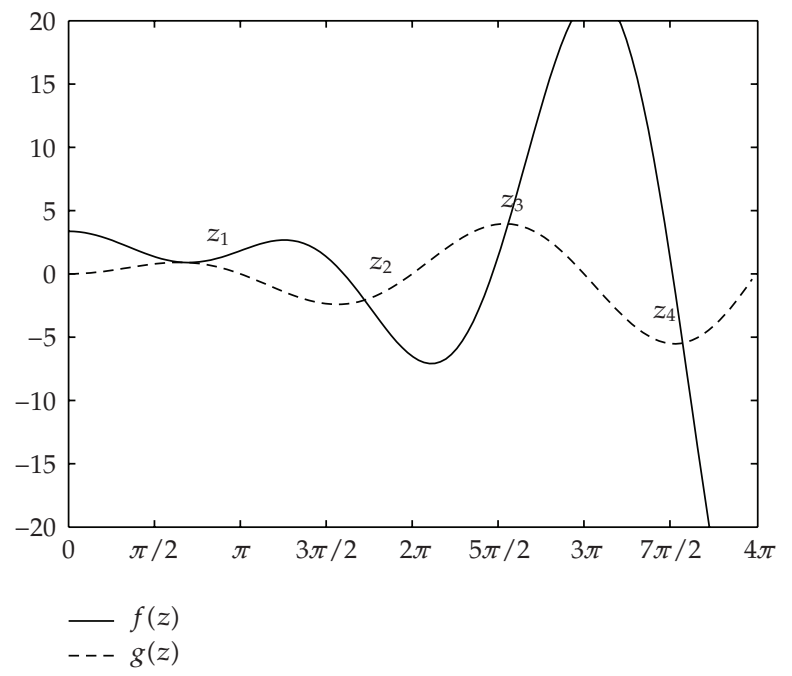

Figure 2: Representation of the curves of $f(z)$ and of $g(z)\left(K=L=a_{1}=1, a_{0}=2, K_{p}=K_{u}=1.5884\right)$ (Case: $K_{p}=K_{u}$ ).

that is, $z_{j}$ verifies

$$
\begin{gathered}
z_{1} \in\left[0, \frac{\pi}{2}\right], \\
z_{j} \in\left[(2 j-3) \frac{\pi}{2},(j-1) \pi\right], \quad \text { for } j \geq 2 .
\end{gathered}
$$

Case $2\left(K_{p} \geq K_{u}\right)$. Figure 2 represents the case where the curves of $f(z)=K K_{u}+\cos (z)\left(a_{0}-\right.$ $\left.\left(z^{2} / L^{2}\right)\right)$ and of $g(z)$ are tangent in $[0, \pi]$, where $K_{u}$ is the largest number of $K_{p}$. 
The plot in Figure 3 corresponds to the case where $K_{p}>K_{u}$ and the plot of $f(z)$ does not intersect $g(z)$ twice in the interval $[0, \pi]$.

Theorem 2.2 is used to verify that $\delta_{i}(z)$ possess only simple roots. By replacing Ls by $s_{1}$ in $(2.2)$, we rewrite $\delta^{*}(s)$ as follows:

$$
\begin{aligned}
\delta^{*}(s) & =e^{L s} \delta(s)=e^{s_{1}} \delta\left(s_{1}\right) \\
& =e^{s_{1}}\left(\left(\frac{s_{1}}{L}\right)^{3}+a_{1}\left(\frac{s_{1}}{L}\right)^{2}+a_{0} \frac{s_{1}}{L}\right)+K\left(K_{p} \frac{s_{1}}{L}+K_{i}\right)
\end{aligned}
$$

For this new quasi-polynomial, we see that $M=3$ and $N=1$ where $M$ and $N$ designate the highest powers of $s$ and $e^{s}$ which appear in $\delta^{*}(s)$. We choose $\eta=\pi / 4$ that satisfies the condition giving by theorem 3 as $\delta_{r}(\eta) \neq 0$ and $\delta_{i}(\eta) \neq 0$. According to Figure 1, we notice that for $-a_{0} / K<K_{p}<K_{u}, \delta_{i}(z)$ possess four roots in the interval $[0,2 \pi-\pi / 4]=[0,7 \pi / 4]$ including the root at origin. As $\delta_{i}(z)$ is odd function of $z$, so it possesses seven roots in $[-2 \pi+$ $\pi / 4,2 \pi-\pi / 4]=[-7 \pi / 4,7 \pi / 4]$. Hence, we can affirm that $\delta_{i}(z)$ has exactly $4 N+M=7$ in $[-2 \pi+\pi / 4,2 \pi+\pi / 4]=[-7 \pi / 4,9 \pi / 4]$. In addition, it can be shown that $\delta_{i}(z)$ has two real roots in each of the intervals $[2 l \pi+\pi / 4,2(l+1) \pi+\pi / 4]$ and $[-2(l+1) \pi+\pi / 4,-2 l \pi+\pi / 4]$ for $l=1,2, \ldots$. It fallows that $\delta_{i}(z)$ has exactly $4 l N+M$ real roots in $[-2 l \pi+\pi / 4,2 l \pi+\pi / 4]$ for $-a_{0} / K<K_{p}<K_{u}$. At the end, according to Theorem 2.2, $\delta_{i}(z)$ has only real roots for every $K_{p}$ in $\left[-a_{0} / K, K_{u}\right]$. For $K_{p} \geq K_{u}$, corresponding to Figures 2 and 3, the roots of $\delta_{i}(z)$ are not real. We pass to determine the superior value of $K_{p}$. According to the definition of $K_{u}$, if $K_{p}=K_{u}$, then the curves of $f(z)$ and $g(z)$ are tangent in the point $\alpha$. Which is translated by

$$
\begin{aligned}
& K K_{u}+\cos (\alpha)\left(a_{0}-\frac{\alpha^{2}}{L^{2}}\right)=a_{1} \frac{\alpha}{L} \sin (\alpha) \\
& \frac{d}{d z}\left[K K_{u}+\cos (z)\left(a_{0}-\frac{z^{2}}{L^{2}}\right)\right]_{z=\alpha} \\
& \quad=\frac{d}{d z}\left[a_{1} \frac{z}{L} \sin (z)\right]_{z=\alpha^{\prime}} \\
& \Longrightarrow-2 \alpha \cos (\alpha)\left(1+a_{1} L\right)+\sin (\alpha)\left(\alpha^{2}-a_{0} L^{2}-a_{1} L\right)=0 \\
& \Longrightarrow \tan (\alpha)=\frac{\alpha\left(2+a_{1} L\right)}{\left(\alpha^{2}-a_{0} L^{2}-a_{1} L\right)}
\end{aligned}
$$

once $\alpha$ is determined, the parameter $K_{u}$ is expressed by the relation:

$$
K_{u}=\frac{1}{K}\left(a_{1} \frac{\alpha}{L} \sin (\alpha)-\cos (\alpha)\left(a_{0}-\frac{\alpha^{2}}{L^{2}}\right)\right) .
$$




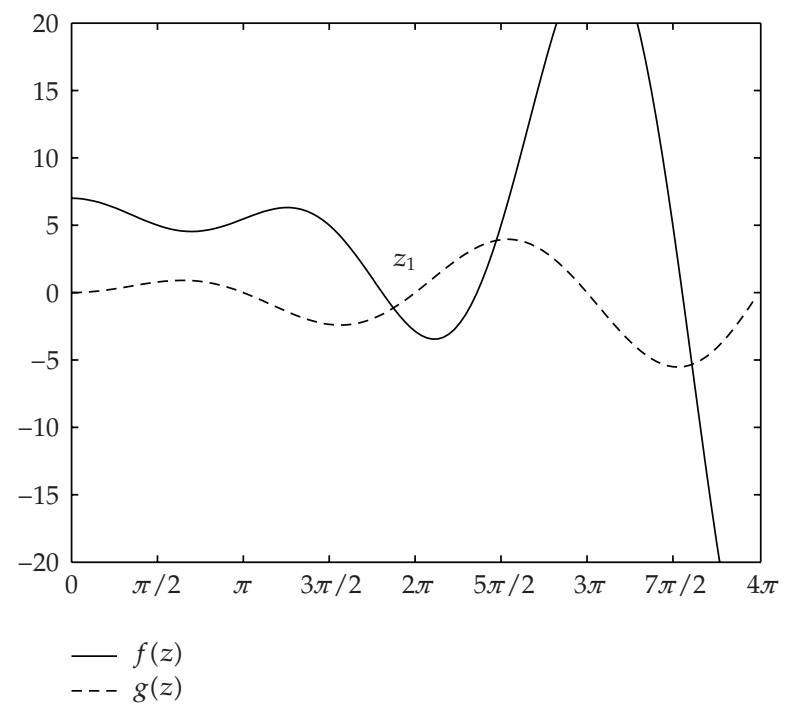

Figure 3: Representation of the curves of $f(z)$ and of $g(z)\left(K=L=a_{1}=1, a_{0}=2, K_{p}=2\right.$, and $K_{u}=1.5884$ (Case: $K_{p}>K_{u}$ ).

Theorem 3.1. The $\delta_{i}(z)$ have real roots if and only if:

$$
-\frac{a_{0}}{K}<K_{p}<\frac{1}{K}\left(a_{1} \frac{\alpha}{L} \sin (\alpha)-\cos (\alpha)\left(a_{0}-\frac{\alpha^{2}}{L^{2}}\right)\right),
$$

where $\alpha$ is the solution of the equation $\tan (\alpha)=\alpha\left(2+a_{1} L\right) /\left(\alpha^{2}-a_{1} L-a_{0} L^{2}\right)$ in the interval $[0, \pi]$.

After determination of the roots of the imaginary part $\delta_{i}(z)$, we pass to the evaluation of these roots by the real part $\delta_{r}(z)$

$$
\begin{aligned}
\delta_{r}(z) & =K K_{i}-K K_{d} \frac{z^{2}}{L^{2}}+\sin (z)\left(\frac{z^{3}}{L^{3}}-a_{0} \frac{z}{L}\right)-a_{1} \frac{z^{2}}{L^{2}} \cos (z) \\
& =K \frac{z^{2}}{L^{2}}\left[-K_{d}+K_{i} \frac{L^{2}}{z^{2}}+\frac{L}{K z}\left[-a_{1} \frac{z}{L} \cos (z)+\sin (z)\left(\frac{z^{2}}{L^{2}}-a_{0}\right)\right]\right] \\
& =K \frac{z^{2}}{L^{2}}\left[-K_{d}+m(z) K_{i}+b(z)\right],
\end{aligned}
$$

where:

$$
\begin{aligned}
& m(z)=\frac{L^{2}}{z^{2}} \\
& b(z)=\frac{L}{K z}\left[-a_{1} \frac{z}{L} \cos (z)+\sin (z)\left(\frac{z^{2}}{L^{2}}-a_{0}\right)\right] .
\end{aligned}
$$


From condition 1 of Theorem 2.1, the roots of $\delta_{r}(z)$ and $\delta_{i}(z)$ have to interlace for $\delta^{*}(s)$ to be stable. We evaluate $\delta_{r}(z)$ at the roots of the imaginary part $\delta_{i}(z)$.

For $z_{0}=0$, we have

$$
\delta_{r}\left(z_{0}\right)=K K_{i}>0,
$$

for $z_{j} \neq z_{0}$, where $j=1,2,3, \ldots$, we get:

$$
\begin{aligned}
\delta_{r}\left(z_{j}\right) & =K \frac{z_{j}^{2}}{L^{2}}\left[-K_{d}+m\left(z_{j}\right) K_{i}+b\left(z_{j}\right)\right] \\
& =K \frac{z_{j}^{2}}{L^{2}}\left[-K_{d}+m_{j} K_{i}+b_{j}\right],
\end{aligned}
$$

where:

$$
\begin{aligned}
& m\left(z_{j}\right)=m_{j}, \\
& b\left(z_{j}\right)=b_{j} .
\end{aligned}
$$

Interlacing the roots of $\delta_{r}(z)$ and $\delta_{i}(z)$ is equivalent to $\delta_{r}\left(z_{0}\right)>0$ (since $\left.K_{i}>0\right), \delta_{r}\left(z_{1}\right)<$ $0, \delta_{r}\left(z_{2}\right)>0 \ldots$

We can use the interlacing property and do it as $\delta_{i}(z)$ which has only real roots to reach that $\delta_{r}(z)$ possess real roots too.

From the previous equations we get the following inequalities:

$$
\left\{\begin{array} { l } 
{ \delta _ { r } ( z _ { 0 } ) > 0 } \\
{ \delta _ { r } ( z _ { 1 } ) < 0 } \\
{ \delta _ { r } ( z _ { 2 } ) > 0 } \\
{ \delta _ { r } ( z _ { 3 } ) < 0 } \\
{ \delta _ { r } ( z _ { 4 } ) > 0 } \\
{ \vdots }
\end{array} \Longrightarrow \left\{\begin{array}{l}
K_{i}>0 \\
K_{d}>m_{1} K_{i}+b_{1} \\
K_{d}<m_{2} K_{i}+b_{2} \\
K_{d}>m_{3} K_{i}+b_{3} \\
K_{d}<m_{4} K_{i}+b_{4} \\
\vdots
\end{array}\right.\right.
$$

Thus intersecting all these regions in the $\left(K_{i}, K_{d}\right)$ space, we get the set of $\left(K_{i}, K_{d}\right)$ values for which the roots of $\delta_{r}(z)$ and $\delta_{i}(z)$ interlace for a fixed value of $K_{p}$. We notice that all these regions are half planes with their boundaries being lines with positive slopes $m_{j}$.

Example 3.2. Consider the plant given by relation (3.1) with the following parameters $K=$ $L=a_{1}=1$ and $a_{0}=2$ :

$$
G(s)=\frac{1}{s^{2}+s+2} e^{-s} .
$$

The imaginary part $\delta_{i}(z)$ has only simple real roots if and only if $-1<K_{p}<1.58$. 
We set the controller parameter $K_{p}$ to 1.3 , which is inside the previous range. For this $K_{p}, \delta_{i}(z)$ takes the form:

$$
z\left(1.3+\cos (z)\left(2-z^{2}\right)-z \sin (z)\right)=0
$$

We next compute some of the positive real roots of this equation and arrange them in increasing order of magnitude:

$$
\begin{gathered}
z_{0}=0, \quad z_{1}=1.3608, \quad z_{2}=1.8905, \quad z_{3}=4.9829, \\
z_{4}=7.9619, \quad z_{5}=11.0976, \quad z_{6}=14.2017
\end{gathered}
$$

Using (3.18) we calculate the parameters $m_{j}$ and $b_{j}$ for $j=1, \ldots, 6$ :

$$
\left\{\begin{array} { l } 
{ m _ { 1 } = 0 . 5 4 , } \\
{ m _ { 2 } = 0 . 2 7 9 8 , } \\
{ m _ { 3 } = 0 . 0 4 0 3 , } \\
{ m _ { 4 } = 0 . 0 1 5 8 , }
\end{array} \quad \left\{\begin{array}{l}
b_{1}=0.54 \\
b_{2}=-0.3150 \\
b_{3}=1.1047 \\
b_{4}=-4.6822
\end{array}\right.\right.
$$

Interlacing the roots of the real and the imaginary part occurs for $K_{p}=1.3$, if and only if the following inequalities are satisfied:

$$
\begin{aligned}
& K_{i}>0, \\
& K_{d}>0.54 K_{i}-0.3150, \\
& K_{d}<0.2798 K_{i}+1.1047, \\
& K_{d}>0.0403 K_{i}-4.6822, \\
& K_{d}<0.0158 K_{i}+7.7736 .
\end{aligned}
$$

The boundaries of these regions are illustrated in Figure 4.

In this case, the stability region is defined by only two boundaries:

$$
\begin{aligned}
& K_{d}=\mathrm{m}_{1} K_{i}+b_{1}, \\
& K_{d}=\mathrm{m}_{2} K_{i}+b_{2},
\end{aligned}
$$




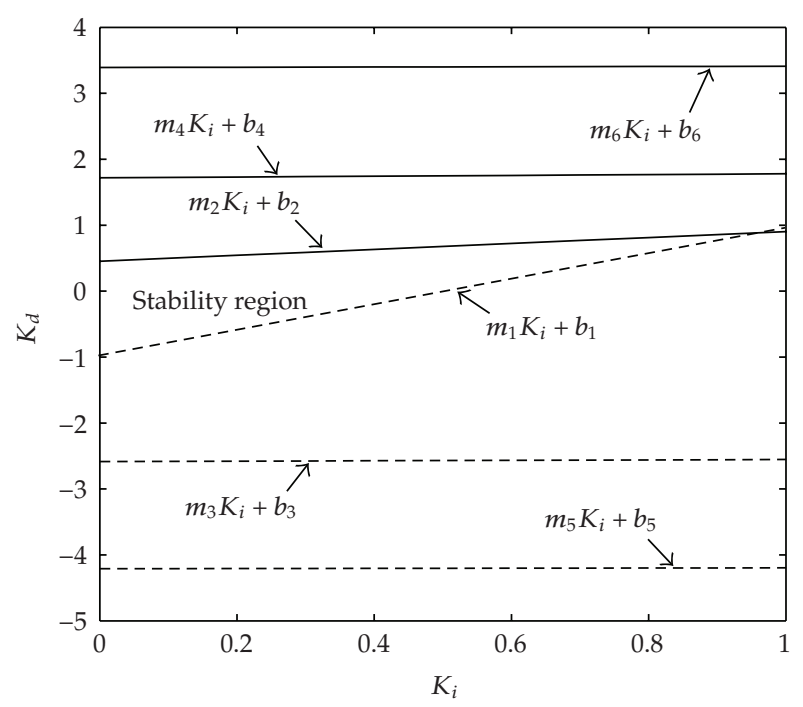

Figure 4: Region boundaries of Example 3.2.

because we have the following inequalities:

$$
\begin{aligned}
& b_{j}<b_{j+2}, \quad \text { for } j=2,4,6, \ldots, \\
& b_{j}>b_{j+2}, \quad \text { for } j=1,3,5,7, \ldots, \\
& m_{j}<m_{j+2}, \quad \text { for } j \geq 1 .
\end{aligned}
$$

Example 3.3. Consider the plant (3.1) with the following parameters $K=2, a_{0}=3, a_{1}=1$, $L=2$ :

$$
G(s)=\frac{2}{s^{2}+s+3} e^{-2 s}
$$

The imaginary part $\delta_{i}(z)$ has only simple real roots if and only if $-1.5<K_{p}<0.98$.

We now set the controller parameter $K_{p}$ to 0.5 , which is inside the previous range. For this $K_{p}, \delta_{i}(z)$ takes the form

$$
z\left(0.5+\cos (z)\left(2-\frac{z^{2}}{4}\right)-0.5 z \sin (z)\right)=0
$$

We next compute some of the positive real roots of this equation and arrange them in increasing order of magnitude

$$
\begin{gathered}
z_{0}=0, \quad z_{1}=1.6480, \quad z_{2}=2.9830, \quad z_{3}=5.4560, \quad z_{4}=8.077, \\
z_{5}=11.22, \quad z_{6}=14.26, \quad z_{7}=17.41 .
\end{gathered}
$$


Using (3.18), we calculate the parameters $m_{j}$ and $b_{j}$ for $j=1, \ldots, 7$ :

$$
\left\{\begin{array} { l } 
{ m _ { 1 } = 1 . 4 7 2 8 , } \\
{ m _ { 2 } = 0 . 4 4 9 5 , } \\
{ m _ { 3 } = 0 . 1 3 4 4 , } \\
{ m _ { 4 } = 0 . 0 6 1 3 , } \\
{ m _ { 5 } = 0 . 0 3 1 8 , } \\
{ m _ { 6 } = 0 . 0 1 9 7 , } \\
{ m _ { 7 } = 0 . 0 1 3 2 , }
\end{array} \quad \left\{\begin{array}{l}
b_{1}=-1.3656 \\
b_{2}=0.4527 \\
b_{3}=-0.9377 \\
b_{4}=1.7176 \\
b_{5}=-2.5853 \\
b_{6}=3.3906 \\
b_{7}=-4.2097
\end{array}\right.\right.
$$

Interlacing the roots of the real and imaginary part occurs for $K_{p}=0.5$, if and only if the following inequalities are satisfied:

$$
\begin{aligned}
K_{i} & >0, \\
K_{d} & >1.4728 K_{i}-1.3656, \\
K_{d} & <0.4495 K_{i}+0.4527, \\
K_{d} & >0.1344 K_{i}-0.9377, \\
K_{d} & <0.0613 K_{i}+1.7176, \\
K_{d} & >0.0318 K_{i}-2.5853, \\
K_{d} & <0.0197 K_{i}+3.3906, \\
K_{d} & >0.0132 K_{i}-4.2097 .
\end{aligned}
$$

The boundaries of these regions are illustrated in Figure 5 .

In this case, the stability region is defined by only two boundaries:

$$
\begin{aligned}
& K_{d}=m_{1} K_{i}+b_{1}, \\
& K_{d}=m_{2} K_{i}+b_{2}, \\
& K_{d}=m_{3} K_{i}+b_{3},
\end{aligned}
$$

because we have the following inequalities:

$$
\begin{aligned}
& b_{j}<b_{j+2,}, \quad \text { for } j=2,4,6, \ldots, \\
& b_{j}>b_{j+2}, \quad \text { for } j=3,5,7, \ldots, \\
& b_{1}<b_{3}, \\
& m_{j}<m_{j+2}, \quad \text { for } j \geq 1 .
\end{aligned}
$$




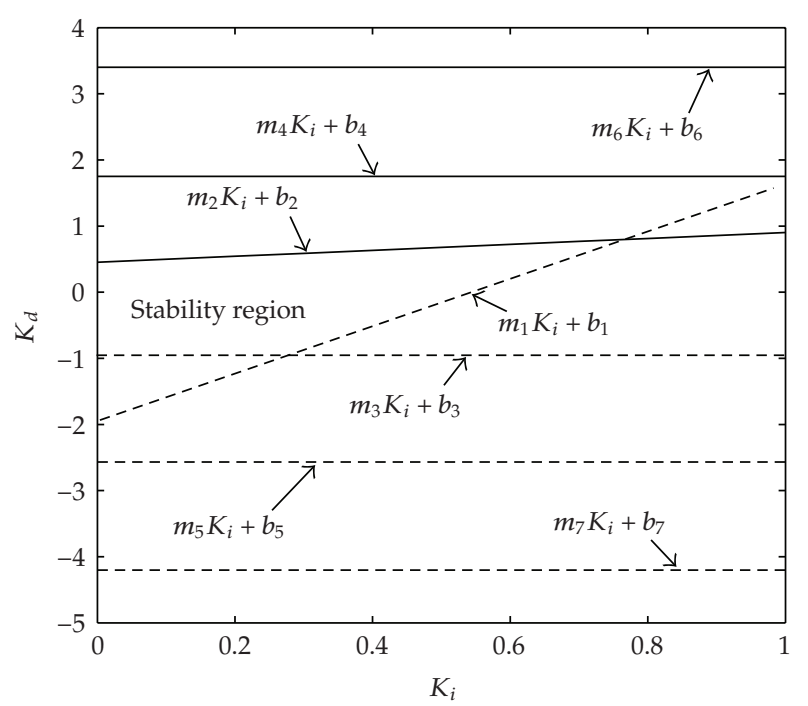

Figure 5: Region boundaries of Example 3.3.

As pointed out in Examples 3.2 and 3.3, the inequalities given by (3.22) represent half planes in the space of $K_{i}$ and $K_{d}$. Their boundaries are given by lines with the following equations:

$$
K_{d}=m_{j} K_{i}+b_{j}, \quad \text { for } j=1,2,3, \ldots
$$

We notice that the difference between Examples 3.2 and 3.3 resides in change of the sign of $\left(b_{1}-b_{3}\right)$ which is explained by the localization of $z_{1}$ and $z_{3}$ in different intervals according to (3.12).

We now state an important technical lemma that allows us to develop an algorithm for solving the PID stabilization problem. This lemma shows the behavior of the parameter $b_{j}, j=$ $2,3,4, \ldots$ for different values of the parameter $K_{p}$ inside the range proposed by Theorem 3.1.

Lemma 3.4. If

$$
-\frac{a_{0}}{K}<K_{p}<\frac{1}{K}\left(a_{1} \frac{\alpha}{L} \sin (\alpha)-\cos (\alpha)\left(a_{0}-\frac{\alpha^{2}}{L^{2}}\right)\right),
$$

where $\alpha$ is the solution of the equation $\tan (\alpha)=\alpha\left(2+a_{1} L\right) /\left(\alpha^{2}-a_{1} L-a_{0} L^{2}\right)$ in the interval $[0, \pi]$.Then:

(1) $b_{j}<b_{j+2}$ for $j=2,4,6, \ldots$,

(2) $b_{j}>b_{j+2}$ for $j=3,5,7, \ldots$,

(3) $m_{j}>m_{j+2}$ for $j \geq 1$, and $\lim _{j \rightarrow \infty} m_{j}=0$. 
Proof. From (3.18) we have

$$
m\left(z_{j}\right)=\frac{L^{2}}{z_{j}^{2}}, \quad 0<z_{j}<z_{j+2} \Longrightarrow \frac{L^{2}}{z_{j}^{2}}>\frac{L^{2}}{z_{j+2}^{2}}, \quad \text { for } j \geq 1
$$

then

$$
m\left(z_{j}\right)>m\left(z_{j+2}\right), \quad \lim _{j \rightarrow \infty} m\left(z_{j}\right)=0 .
$$

Remark 3.5. As we can see from Figure 1 , the odd roots of $\delta_{i}(z)$, that is, $z_{j}$ for $j=3,5,7, \ldots$, verify $z_{j} \in[(2 j-3) \pi / 2,(j-1) \pi]$ and are getting closer to $(2 j-3) \pi / 2$ as $j$ increase, so we have:

$$
\lim _{j \rightarrow \infty} \cos \left(z_{j}\right)=0, \quad \lim _{j \rightarrow \infty} \sin \left(z_{j}\right)=-1
$$

Moreover, since cosine function and sine function are monotonically increasing between $(2 j-$ 3) $\pi / 2$ and $(j-1) \pi$, we have

$$
\left\{\begin{array} { l } 
{ \operatorname { c o s } ( z _ { 3 } ) > \operatorname { c o s } ( z _ { 5 } ) > \operatorname { c o s } ( z _ { 7 } ) > \cdots } \\
{ \operatorname { s i n } ( z _ { 3 } ) > \operatorname { s i n } ( z _ { 5 } ) > \operatorname { s i n } ( z _ { 7 } ) > \cdots }
\end{array} \Longrightarrow \left\{\begin{array}{l}
\cos \left(z_{j}\right)>\cos \left(z_{j+2}\right)>0, \\
\sin \left(z_{j+2}\right)<\sin \left(z_{j}\right)<0 .
\end{array}\right.\right.
$$

Remark 3.6. As we can see from Figure 1, the even roots of $\delta_{i}(z)$ that is, $z_{j}$ for $j=2,4,6, \ldots$ verify $z_{j} \in[(2 j-3) \pi / 2,(j-1) \pi]$ and are getting closer to $(2 j-3) \pi / 2$ as $j$ increase, so we have:

$$
\lim _{j \rightarrow \infty} \cos \left(z_{j}\right)=0, \quad \lim _{j \rightarrow \infty} \sin \left(z_{j}\right)=1 .
$$

Moreover, since cosine function and sine function are monotonically decreasing between $(2 j-$ 3) $\pi / 2$ and $(j-1) \pi$, we have:

$$
\left\{\begin{array} { l } 
{ \operatorname { c o s } ( z _ { 2 } ) < \operatorname { c o s } ( z _ { 4 } ) < \operatorname { c o s } ( z _ { 6 } ) < \cdots } \\
{ \operatorname { s i n } ( z _ { 2 } ) < \operatorname { s i n } ( z _ { 4 } ) < \operatorname { s i n } ( z _ { 6 } ) < \cdots }
\end{array} \Longrightarrow \left\{\begin{array}{l}
\cos \left(z_{j}\right)<\cos \left(z_{j+2}\right)<0, \\
0<\sin \left(z_{j}\right)<\sin \left(z_{j+2}\right) .
\end{array}\right.\right.
$$

The change of $b\left(z_{j}\right)$ can be found with graphical approach. Given a stabilizing $K_{p}$ value inside the admissible range by using Theorem 3.7, the curve of $\delta_{i}(z)$ is plotted to obtain their roots denoted by $z_{j}, j=1,2,3, \ldots$, the curves of $\left(-a_{1}(z / L)\right) \cos (z)$ and $\sin (z)\left(z^{2} / L^{2}-a_{0}\right)$ are plotted too in order to understand the behavior of $b\left(z_{j}\right)$.

As far as the odd roots of $\delta_{i}(z)$ are concerned, the corresponding $\sin (z)\left(z^{2} / L^{2}-a_{0}\right)$ is decreasing by large magnitude, and for the even ones, the corresponding $\sin (z)\left(z^{2} / L^{2}-\right.$ $\left.a_{0}\right)$ is increasing by large magnitude. However, compared to the change of $\sin \left(z_{j}\right)\left(z_{j}^{2} / L^{2}-\right.$ $\left.a_{0}\right)$, the difference between the values of $\left(-a_{1}\left(z_{j+2} / L\right)\right) \cos \left(z_{j+2}\right)$ and $\left(-a_{1}\left(z_{j} / L\right)\right) \cos \left(z_{j}\right)$ is 


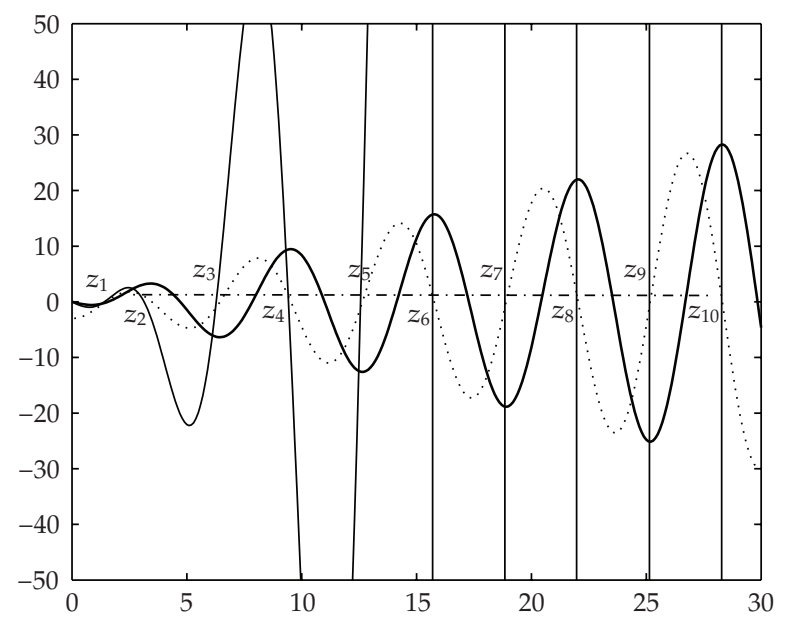

Figure 6: Plots of $\delta_{i}(z)$ (dotted line), $\left(-a_{1}(z / L)\right) \cos (z)$ (thick solid line) and $\sin (z)\left(z^{2} / L^{2}-a_{0}\right)$ (thin solid line).

much smaller than both for odd and even $j$. Thus, the $b\left(z_{j}\right)$ have the similar change rules as $\sin \left(z_{j}\right)\left(z_{j}^{2} / L^{2}-a_{0}\right)$,

$$
\begin{aligned}
b_{j+2}-b_{j}= & \frac{L}{K}\left[-a_{1} \frac{z_{j+2}}{L} \cos \left(z_{j+2}\right)+\sin \left(z_{j+2}\right)\left(\frac{z_{j+2}^{2}}{L^{2}}-a_{0}\right)\right] \\
& -\frac{L}{K}\left[-a_{1} \frac{z_{j}}{L} \cos \left(z_{j}\right)+\sin \left(z_{j}\right)\left(\frac{z_{j}^{2}}{L^{2}}-a_{0}\right)\right], \\
b_{j+2}-b_{j}= & \frac{a_{1}}{L}\left[\cos \left(z_{j}\right)-\cos \left(z_{j+2}\right)\right] \\
& +\frac{L}{K}\left[\frac{\sin \left(z_{j+2}\right)}{z_{j+2}}\left(\frac{z_{j+2}^{2}}{L^{2}}-a_{0}\right)-\frac{\sin \left(z_{j}\right)}{z_{j}}\left(\frac{z_{j}^{2}}{L^{2}}-a_{0}\right)\right] .
\end{aligned}
$$

Using Remarks 3.5 and 3.6, we can affirm that

(i) $b_{j}>b_{j+2}$ and $b_{j} \rightarrow-\infty$ as $j \rightarrow+\infty$ for odd values of $j$,

(ii) $b_{j}<b_{j+2}$ and $b_{j} \rightarrow+\infty$ as $j \rightarrow+\infty$ for even values of $j$.

We are ready to state the main results of our work.

Theorem 3.7. Under the above assumptions on $K, L, a_{0}$, and $a_{1}$, the range of $K_{p}$ values for which a solution exists to the PID stabilization problem of an open-loop stable plant with transfer function $G(s)$ is given by

$$
-\frac{a_{0}}{K}<K_{p}<\frac{1}{K}\left(a_{1} \frac{\alpha}{L} \sin (\alpha)-\cos (\alpha)\left(a_{0}-\frac{\alpha^{2}}{L^{2}}\right)\right)
$$

where $\alpha$ is the solution of the equation $\tan (\alpha)=\alpha\left(2+a_{1} L\right) /\left(\alpha^{2}-a_{1} L-a_{0} L^{2}\right)$ in the interval $[0, \pi]$. 


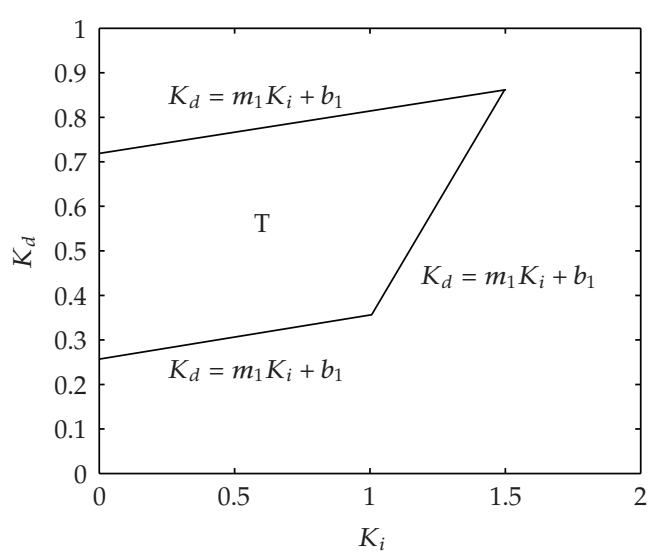

(a)

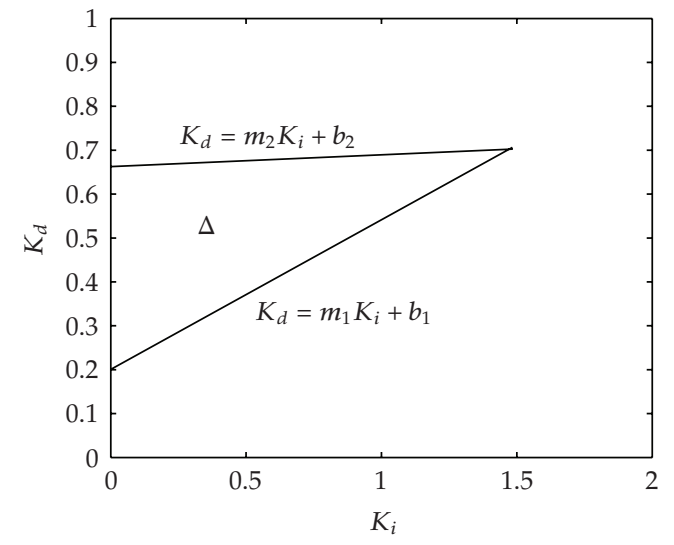

(b)

Figure 7: The stabilizing region of $\left(K_{p}, K_{i}\right),(\mathrm{a}): b_{3}>b_{1},(\mathrm{~b}): b_{1}>b_{3}$.

For $K_{p}$ values outside this range, there are no stabilizing PID controllers. The complete stabilizing region is given by

(1) the cross-section of the stabilizing region in the $\left(K_{i}, K_{d}\right)$ space is the Trapezoid $\mathrm{T}$ if $b_{3}>b_{1}$,

(2) the cross-section of the stabilizing region in the $\left(K_{i}, K_{d}\right)$ space is the triangle $\Delta$ if $b_{1}>b_{3}$,

The parameters $b_{j}, m_{j}, j=1,2,3$ necessary for determining the boundaries, can be determined using (3.21) where $z_{j}, j=1,2,3$ are the positive-real solutions of $\delta_{i}(z)$ arranged in ascending order of magnitude.

In view of Theorem 3.7, we propose an algorithm to determine the set of all stabilizing parameters for a second order delay system.

An algorithm for determining PID parameters is given as follows

(1) Choose $K_{p}$ in the interval suggested by Theorem 3.7 and initialize $j=1$,

(2) Find the roots $z_{j}$ of $\delta_{i}(z)$,

(3) Compute the parameter $b_{j}, m_{j}$ associated with the $z_{j}$ for $j=1,2,3$ founded,

(4) Determine the stability region in the plane $\left(K_{i}, K_{d}\right)$ using Figure 7 (Theorem 3.7).

(5) Go to step 1.

\section{Conclusion}

In this work, we have proposed an extension of Hermit-Biehler theorem to compute the stability region for second-order delay system controlled by PID controller. The procedure is based first on determining the range of proportional gain value $K_{p}$ for which a solution to PID stabilization exists. Then, it is shown that for a fixed $K_{p}$ inside this range, the stabilizing integral $K_{i}$ and derivative gain $K_{d}$ values lie inside a region with known shape and boundaries. 


\section{References}

[1] Q. C. Zhong, Robust Control of Time Delay System, Springer, London, UK, 2006.

[2] S.-I. Niculescu, Delay Effects on Stability, vol. 269 of Lecture Notes in Control and Information Sciences, Springer, London, UK, 2001.

[3] C. Knospe, "PID control," IEEE Control Systems Magazine, vol. 26, no. 1, pp. 30-31, 2006.

[4] H. Takatsu, T. Itoh, and M. Araki, "Future needs for the control theory in industries-report and topics of the control technology survey in Japanese industry," Journal of Process Control, vol. 8, no. 5-6, pp. 369-374, 1998.

[5] G. J. Silva, A. Datta, and S. P. Bhattacharyya, PID Controllers for Time-Delay Systems, Control Engineering, Birkhäuser, Boston, Mass, USA, 2005.

[6] R. Toscano, "A simple robust PI/PID controller design via numerical optimization approach," Journal of Process Control, vol. 15, no. 1, pp. 81-88, 2005.

[7] R. Padma Sree, M. N. Srinivas, and M. Chidambaram, "A simple method of tuning PID controllers for stable and unstable FOPTD systems," Computers and Chemical Engineering, vol. 28, no. 11, pp. 22012218, 2004.

[8] C. Hwang and J. H. Hwang, "Stabilisation of first-order plus dead-time unstable processes using PID controllers," IEE Proceedings: Control Theory and Applications, vol. 151, no. 1, pp. 89-94, 2004.

[9] J. McKay, "The D-partition method applied to systems with dead time and distributed lag," Measurement and Control, vol. 3, no. 10, pp. 293-294, 1970.

[10] T. Lee, J. M. Watkins, T. Emami, and S. Sujoldžić, "A unified approach for stabilization of arbitrary order continuous-time and discrete-time transfer functions with time delay using a PID controller," in Proceedings of the IEEE Conference on Decision and Control (CDC '07), pp. 2100-2105, 2007.

[11] L. M. Eriksson and M. Johansson, "PID controller tuning rules for varying time-delay systems," in Proceedings of the American Control Conference, pp. 619-625, July 2007.

[12] S.-J. Li and Y.-X. Liu, "An improved approach to nonlinear dynamical system identification using PID neural networks," International Journal of Nonlinear Sciences and Numerical Simulation, vol. 7, no. 2, pp. 177-182, 2006.

[13] Y. Zhang, F. Wang, Y. Song, Z. Chen, and Z. Yuan, "Recurrent neural networks-based multivariable system PID predictive control," Frontiers of Electrical and Electronic Engineering in China, vol. 2, no. 2, pp. 197-201, 2007.

[14] H. Shu and Y. Pi, "PID neural networks for time-delay systems," Computers and Chemical Engineering, vol. 24, no. 2-7, pp. 859-862, 2000.

[15] H. Shu, X. Guo, and H. Shu, "PID neural networks in multivariable systems," in Proceedings of the IEEE International Symposium on Intelligent Control, pp. 440-444, 2002.

[16] A. Roy and K. Iqbal, "PID controller tuning for the first-order-plus-dead-time process model via Hermite-Biehler theorem," ISA Transactions, vol. 44, no. 3, pp. 363-378, 2005.

[17] G. J. Silva, A. Datta, and S. P. Bhattacharyya, "Stabilization of time delay systems," in Proceedings of the American Control Conference, vol. 2, pp. 963-970, 2000.

[18] G. J. Silva, A. Datta, and S. P. Bhattacharyya, "PI stabilization of first-order systems with time delay," Automatica, vol. 37, no. 12, pp. 2025-2031, 2001.

[19] G. J. Silva, A. Datta, and S. P. Bhattacharyya, "Stabilization of first-order systems with time delay using the PID controller," Proceedings of the American Control Conference, vol. 6, pp. 4650-4655, 2001.

[20] G. J. Silva, A. Datta, and S. P. Bhattacharyya, "New results on the synthesis of PID controllers," IEEE Transactions on Automatic Control, vol. 47, no. 2, pp. 241-252, 2002.

[21] H. Xu, A. Datta, and S. P. Bhattacharyya, "PID stabilization of LTI plants with time-delay," in Proceedings of the 42nd IEEE Conference on Decision and Control (DC '03), vol. 4, pp. 4038-4043, 2003.

[22] V. A. Oliveira, M. C. M. Teixeira, and L. V. Cossi, "Stabilizing a class of time delay systems using the Hermite-Biehler theorem," Linear Algebra and Its Applications, vol. 369, pp. 203-216, 2003.

[23] V. A. Oliveira, L. V. Cossi, A. M. F. Silva, and M. C. M. Teixeira, "PID stabilization of a class of time delay systems," in Proceedings of the 44th IEEE Conference on Decision and Control, and the European Control Conference (CDC-ECC' 05), pp. 1367-1372, 2005.

[24] V. A. Oliveira, L. V. Cossi, M. C. M. Teixeira, and A. M. F. Silva, "Synthesis of PID controllers for a class of time delay systems," Automatica, vol. 45, no. 7, pp. 1778-1782, 2009.

[25] S. P. Bhattacharyya, H. Chapellat, and L. H. Keel, Robust Control: The Parametric Approach, PrenticeHall, Upper Saddle River, NJ, USA, 1995. 


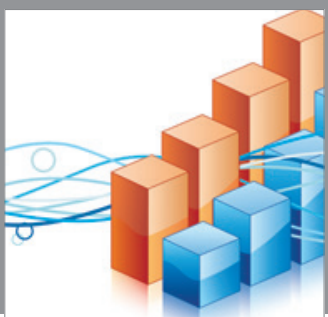

Advances in

Operations Research

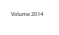

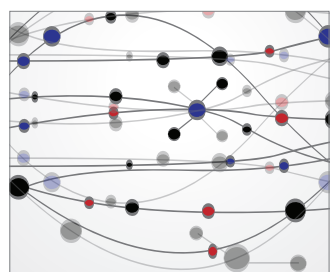

\section{The Scientific} World Journal
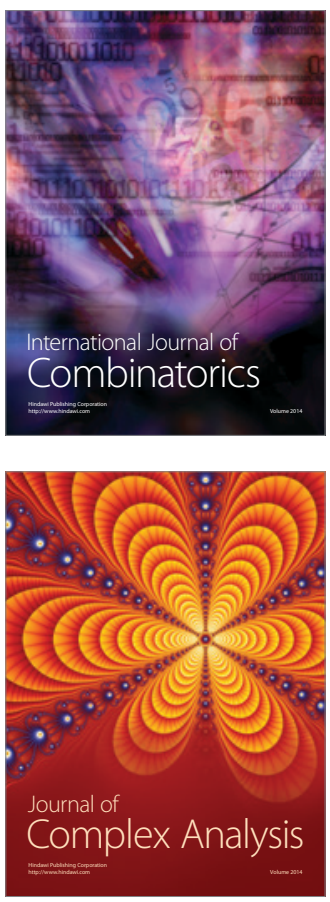

International Journal of

Mathematics and

Mathematical

Sciences
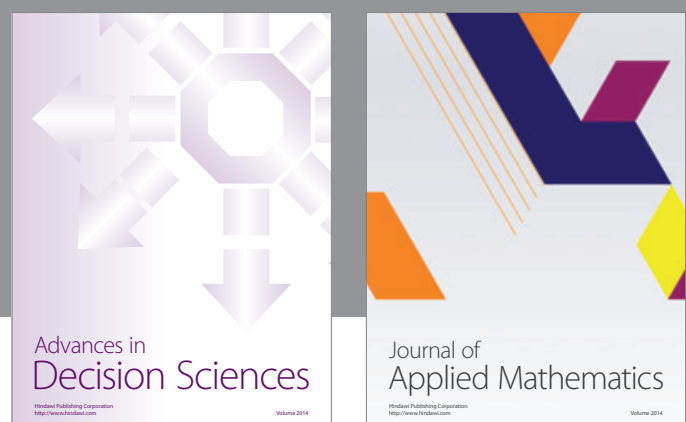

Journal of

Applied Mathematics
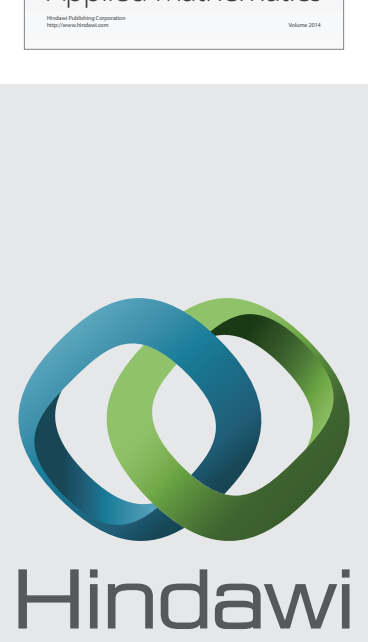

Submit your manuscripts at http://www.hindawi.com
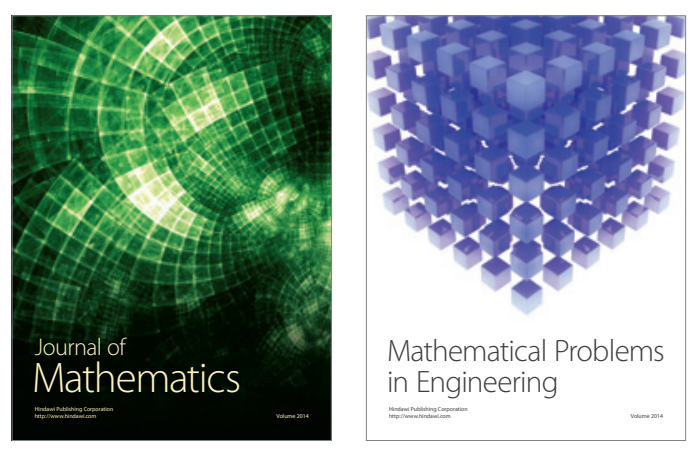

Mathematical Problems in Engineering
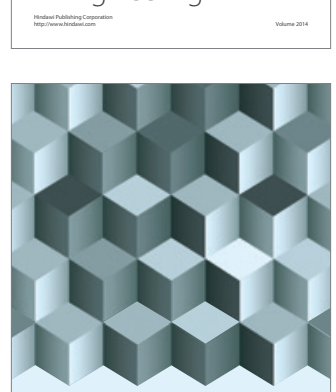

Journal of

Function Spaces
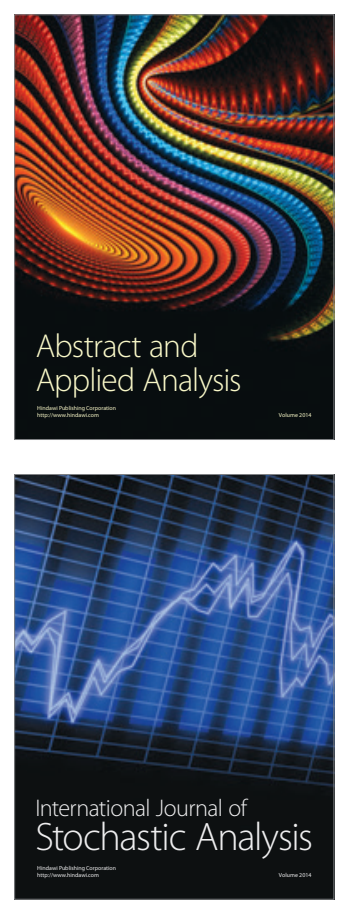

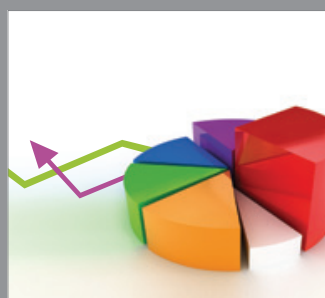

ournal of

Probability and Statistics

Promensencen
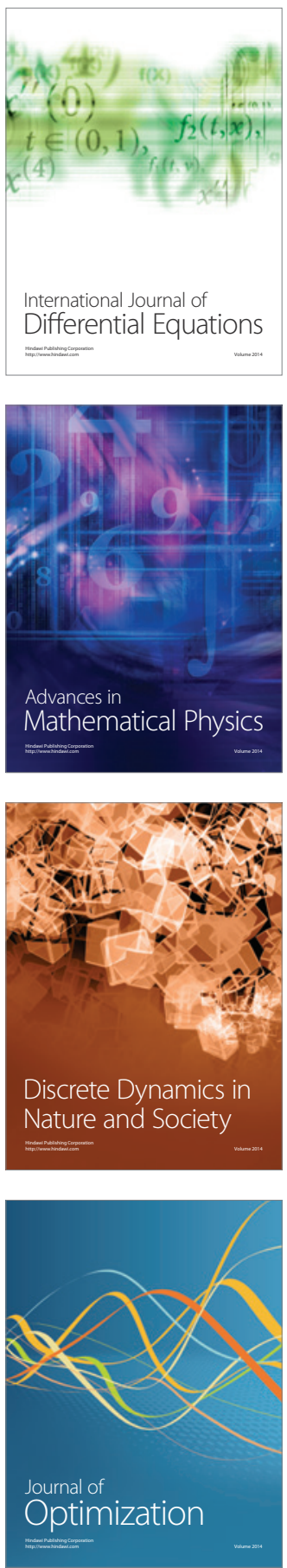